\title{
The Relationship between Cerebral Blood Flow Velocity Fluctuations and Sleep State in Normal Newborns
}

\author{
F. FERRARRI, A. W. R. KELSALL, J. M. RENNIE, AND D. H. EVANS \\ Neonatal Intensive Care Unit, Rosie Maternity Hospital, Cambridge, United Kingdom [A.W.R.K., J.M.R.], \\ Department of Paediatrics, University of Modena, Modena, Italy [F.F.], and Department of Medical Physics,
} University of Leicester, United Kingdom [D.H.E.]

\begin{abstract}
Cerebral blood flow velocity was recorded for an average of 23 4-min epochs during natural sleep in 11 normal full-term newborn babies. Intracranial pressure, core temperature, and respiration were simultaneously and non-invasively monitored. Sleep state was classified using information from EEG, pattern of respiration, and eye and body movements by a trained observer. From a total of 238 epochs, 66 were considered to occur in quiet sleep, 101 in active sleep, and in 77 the baby was awake, in a transitional state or moving excessively. Slow cyclical variations in cerebral blood flow velocity were observed with a frequency of between 2 and 6 cycles/min, and these were of significantly greater amplitude during quiet sleep (24\%) compared to active sleep $(16 \% ; p<0.0001$, Mann Whitney $U$ test). There was no difference in median cerebral blood flow velocity $(7.5 \mathrm{~cm} / \mathrm{s})$. The cyclical variation observed in normal babies were similar to those described in preterm babies and adults, at a similar frequency to $B$ waves in intracranial pressure. They may represent vasomotor waves in the small autoregulatory arteries of the brain. Reduction in sensitivity of the receptors initiating the waves may occur in active sleep or there may be competition from other oscillatory mechanisms. (Pediatr Res 35: 50-54, 1994)

\section{Abbreviations}

CBF, cerebral blood flow

CBFV, cerebral blood flow velocity

ICP, intracranial pressure

CBF is linked to metabolic demand, and it is therefore not surprising that total CBF is about $30 \%$ lower during quiet sleep when compared with active sleep in adult humans (1). CBF measured with jugular occlusion plethysmography was also lower in quiet sleep in term newborns $(2,3)$. No difference in $\mathrm{CBF}$ between sleep states was shown in a group of 15 preterm infants, who have lower CBF than adults and whose cerebral metabolic needs may be less variable between sleep states (4). Using Doppler ultrasound to monitor the velocity of blood flow in the anterior cerebral artery Jorch et al. (5) demonstrated a lower velocity during quiet sleep than active sleep in a group of preterm and term neonates. Doppler ultrasound is unable to quantitate CBF

Received December 1, 1992; accepted August 31, 1993.

Correspondence: Dr. Janet M Rennie, NICU, Rosie Maternity Hospital, Robinson Way, Cambridge, CB2 2SW, UK

A. W. R. K. is an Action Research Fellow. but has the advantage that the method is noninvasive and can give continuous information. The semicontinuous system developed in Leicester $(6,7)$ is particularly suitable for sleep studies as once the small probe is stuck on to the skin of the temporal bone above the middle cerebral artery the infant can be allowed to sleep undisturbed, contrasting with the methods of jugular occlusion plethysmography $(2,3)$ and radioactive $\mathrm{Xe}(4)$ which are likely to alter the sleep state of the infant.

Recently a considerable variation in CBFV occurring with slow, regular cycles with a frequency of between 1 and $5 / \mathrm{min}$ $(0.016-0.08 \mathrm{~Hz})$ has been seen in prolonged Doppler recordings of CBFV from preterm babies $(8,9)$. Slow regular cycles have been seen in the CBFV of adults and thought to be the origin of a similar phenomenon described by Lundberg in intracranial pressure (Lundberg B waves). In a group of adult head-injured patients slow regular cycles in CBFV were completely synchronous with variations in intracranial pressure monitored invasively (10). The cycles were more prominent in conditions in which there was low intracranial compliance, but could be seen in normal individuals where they were more obvious during sleep (11). Slow cyclical variations occurring with a similar frequency (but not always in phase) have long been known to occur in other physiological parameters such as heart rate, respiration, and blood pressure. These are in part due to thermoregulation (12) and the control of vasomotor tone by the autonomic nervous system. Peripheral and central chemoreceptors are less sensitive during active sleep making it possible that there would be differences between slow fluctuations during different sleep states.

This study was designed to investigate changes in CBFV with particular regard to slow cycling during different sleep states in normal healthy term newborns. Information regarding central temperature, respiration, and ICP was collected using noninvasive methods to examine the influence of these physiological variables on the slow cycles in CBFV which we expected to observe.

\section{MATERIALS AND METHODS}

Patient population. The mothers of 11 normal newborn infants gave permission for their infants to be studied after reading an explanatory leaflet containing a photograph of the recording devices in place on a baby, receiving a verbal explanation, and being given time to think and discuss the baby's participation with their partner. Twenty mothers who were approached during the 2-mo period refused, usually because they were trying to establish breast feeding and did not want the baby to be disturbed by the positioning of monitor leads after studying the photograph. The study had prior approval from the Cambridge District Ethical Committee. Mothers of singleton infants were selected at 

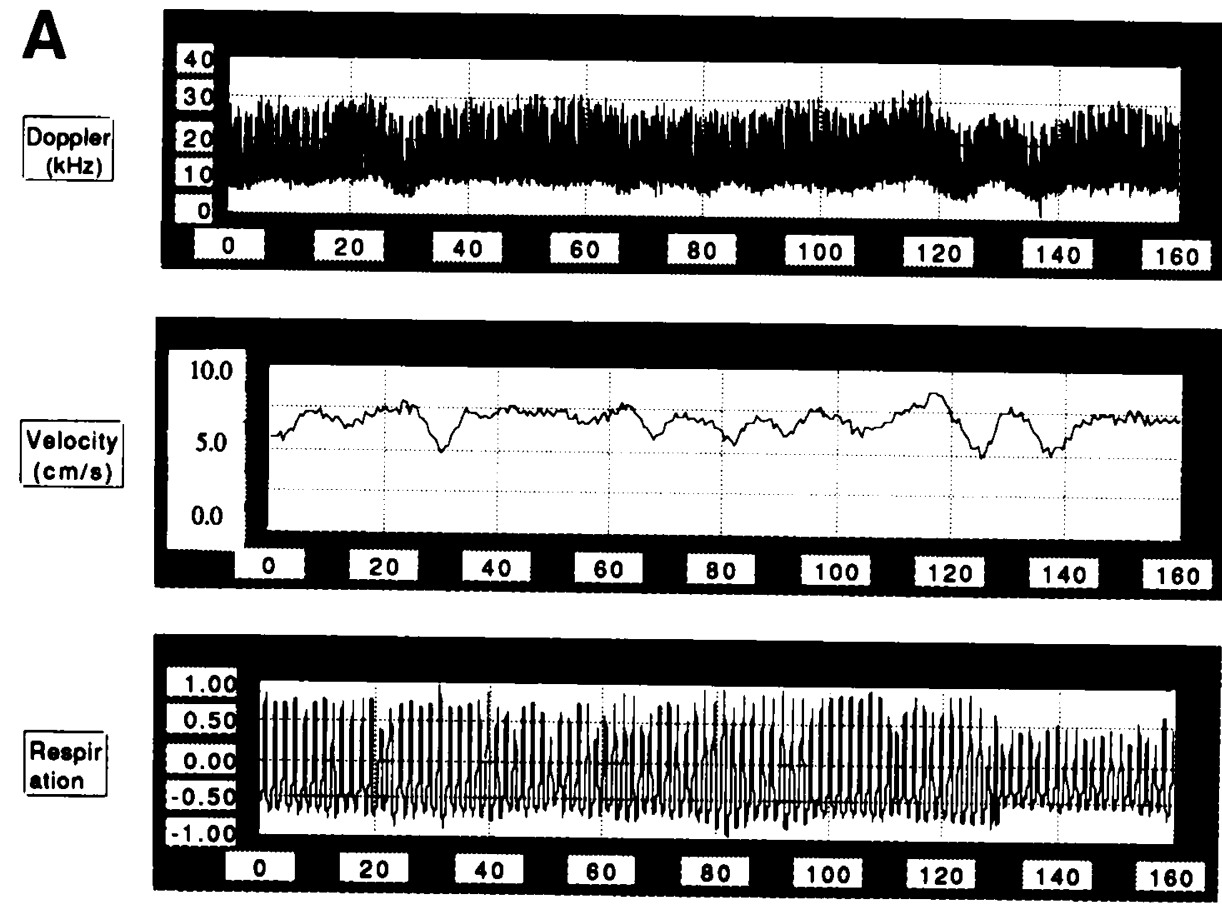

Seconds
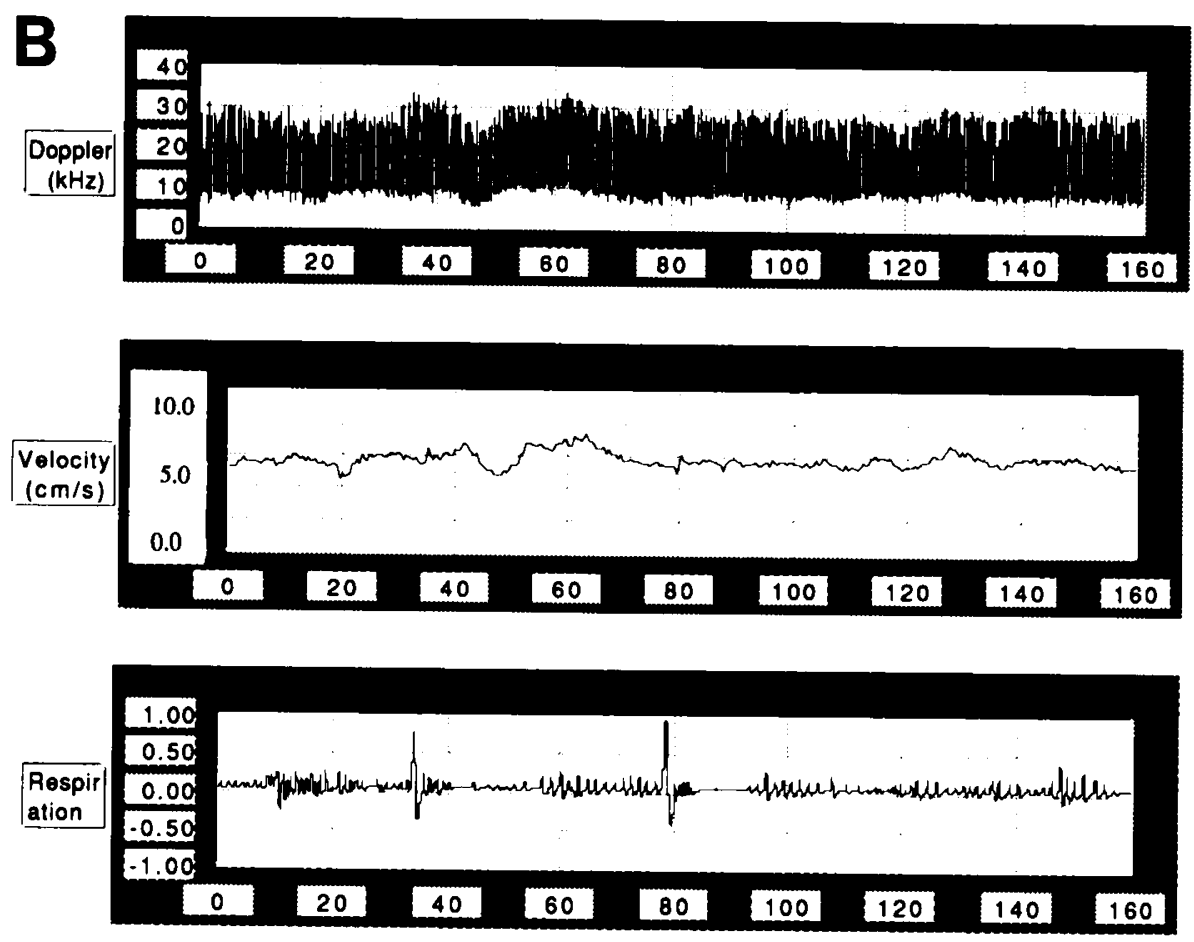

\section{Seconds}

Fig. 1. Example of 4-min recording showing Doppler frequency-shifted signal in channel 1, with beneath it in channel 2 the average velocity calculated and plotted for each heartbeat. Respiratory signal obtained from a monitor is recorded in channel 3. $A$ ) epoch recorded in quiet sleep and $B$ ) in active sleep. Both recordings are from the same baby.

random from those on the postnatal wards at the Rosie Maternity Hospital, Cambridge. The mean gestational age of the babies was 38.5 wk (range $36-41 \mathrm{wk}$ ) with a mean birthweight of $3.33 \mathrm{~kg}$ (range $2.6-4.4 \mathrm{~kg}$ ). Seven were born by normal vaginal vertex delivery and four by caesarean section. The postnatal age at the time of the study ranged between 1 and $9 \mathrm{~d}$ with a mean of 3.8 d. Infants were studied just after a feed and mothers were often present for the whole time it took to site the recording electrodes and complete a cycle of sleep, which was usually about $2 \mathrm{~h}$. Recordings were made in the Neonatal Unit with an investigator (F.F.) present for the duration of the study.

Sleep state. Definition of sleep state was made using the method of Prechtl et al. (13) by a trained observer (F.F.). EEG from four channels was recorded using an Oxford Medilog 
Table 1. Results of comparison of mean CBFV and the frequency and amplitude of slow cycles in CBFV between epochs of quiet and active sleep in normal babies

\begin{tabular}{|c|c|c|c|}
\hline & $\begin{array}{c}\text { Quiet sleep } \\
(n=66 \text { epochs })\end{array}$ & $\begin{array}{c}\text { Active sleep } \\
(n=101 \text { epochs })\end{array}$ & Mann-Whitney $p$ \\
\hline Median CBFV (cm/s) (interquartile range) & $7.5(5.25-9.75)$ & $8(6.75-9.75)$ & 0.42 (NS) \\
\hline Mean cycles/min (range) & $4(2-6)$ & $3.3(2-5)$ & $<0.0001$ \\
\hline Median amplitude of cycles $(\mathrm{cm} / \mathrm{s})$ (IQ range) & $1.5(1.1-2.25)$ & $1.15(0.87-1.37)$ & $<0.0001$ \\
\hline Median amplitude (\%age) (IQ range) & $24 \%(19-30)$ & $16 \%(13-19)$ & $<0.0001$ \\
\hline
\end{tabular}

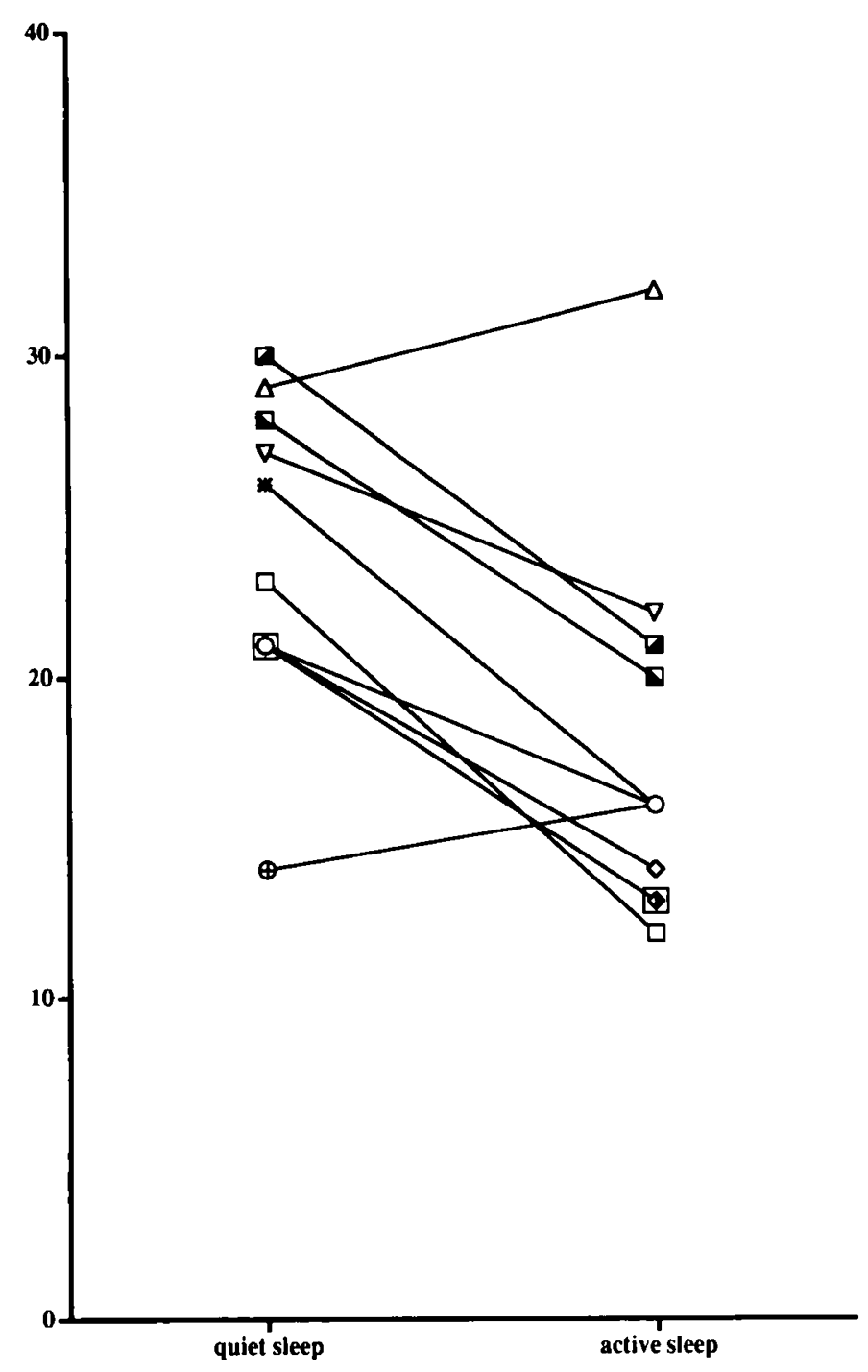

Fig. 2. Percentage change of the cycles seen in CBFV during the 4min epochs, calculated by dividing the maximum depth of a cycle by the predominant velocity present during the epoch. All the epochs collected in quiet sleep and all those in active sleep have been averaged for each baby and the lines connect the results from individuals.

recorder and used together with monitoring of respiration and eye and body movement to classify sleep state. Body movements (present in state 2, active sleep; absent in state 1, quiet sleep) eye movements (present in state 2 absent in state 1 ), opening or closing of the eyes, and crying were used for identification of Prechtl's behavioral states. A 3-min moving window was moved across these variables which had been closely monitored and recorded on paper, and the state was deemed present if throughout the 3-min period its configuration met the criteria required for state 1 or state 2 (14).

$C B F V$. CBFV was measured using the semicontinuous system described by Fenton et al. (7). A small continuous wave Doppler ultrasound probe $0.5 \mathrm{~cm}$ in diameter was fixed over the site of the middle cerebral artery using collodion and adhesive tape. Stomahesive was used to protect the baby's skin. The Doppler signal was processed using the microcomputer based system of Schlindwein et al. (15). This system performs a fast Fourier transform every $6.25 \mathrm{~ms}$ and calculates the peak and intensity weighted mean of the Doppler frequency spectrum. An analog signal representing the peak velocity envelope was passed to a second microcomputer, an Apple Macintosh II running Labview, where it was stored. Recordings lasted for $4 \mathrm{~min}$. CBFV was calculated on a beat-by-beat basis by halving the mean value of the instantaneous maximum velocity over the cardiac cycle (16).

A 4-min epoch taken during quiet sleep is shown in Figure $1 A$. The top tracing is the peak envelope of the Doppler frequency-shifted signal recorded from the middle cerebral artery and the channel beneath shows the mean CBFV calculated as described and plotted separately for each cardiac cycle. The baseline (predominant) velocity for the whole epoch was noted as the velocity present when no cycles were occurring; in this example the velocity was $7 \mathrm{~cm} / \mathrm{s}$. Slow cycles were recorded as present when there was a regular oscillation in velocity causing a change from the baseline velocity of more than $1 \mathrm{~cm} / \mathrm{s}$, with at least two cycles and less than six cycles being present per minute. The classification was done by W. K. who was not involved in making the recordings and was blind to sleep state at the time. The amplitude of the deepest slow cycle present within the 4-min epoch was measured by subtracting the minimum from the maximum velocity, and the percentage amplitude calculated by dividing this value by the baseline velocity referred to earlier.

To validate the visual classification of the presence of slow cycling made by carefully inspecting all the recordings the frequencies present were analysed using fast Fourier transform of the physiological data. This allowed identification of whether or not a peak was present corresponding to the region of interest. Using fast Fourier transformation slow cycling was defined as present if a peak occurred in the CBFV signal between 0.04 and $0.06 \mathrm{~Hz}$ (corresponding to a frequency of between 2 and $5 / \mathrm{min}$ ).

$I C P$ and core temperature. The applanation fontanometer developed in Oxford was used (17). A small plastic air filled blister was fixed to the skin over the fontanelle with collodion. The output from this device was stored within Labview, together with that from a rectal temperature probe and a Graseby dynamics MR 10 respiration monitor. The signals can be seen in the example of a recording given in Figure $1 A$ and $B$.

\section{RESULTS}

An average number of 23 4-min epochs was recorded for each baby (range 17-31), giving 238 epochs for analysis. Seventy-one of these proved unsuitable for further consideration either because the baby was awake, in transitional sleep, or there was movement artifact. Analysis of the remaining 167 epochs from the same 11 subjects (range 11-26/baby) by visual inspection and by fast Fourier transformation as above to evaluate the cycling activity showed that both the amplitude and frequency of slow cycles in CBFV were significantly increased during quiet sleep compared to active sleep (Fig. $1, A$ and $B$; Table 1). Within subjects the average percentage amplitude of the slow cycles from epochs of quiet sleep was greater than that from active sleep in 9 of the 11 babies (Fig. 2). The amplitude difference was readily 


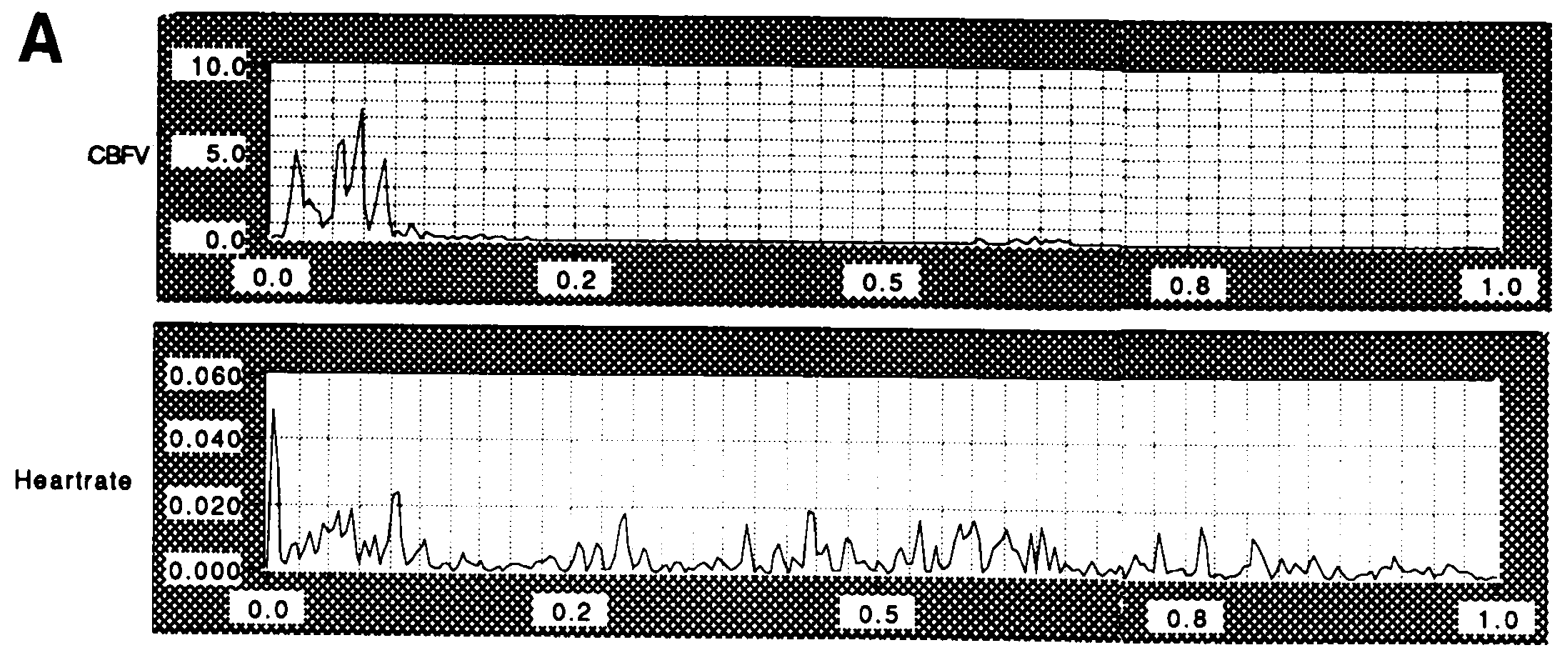

$\mathrm{Hz}$

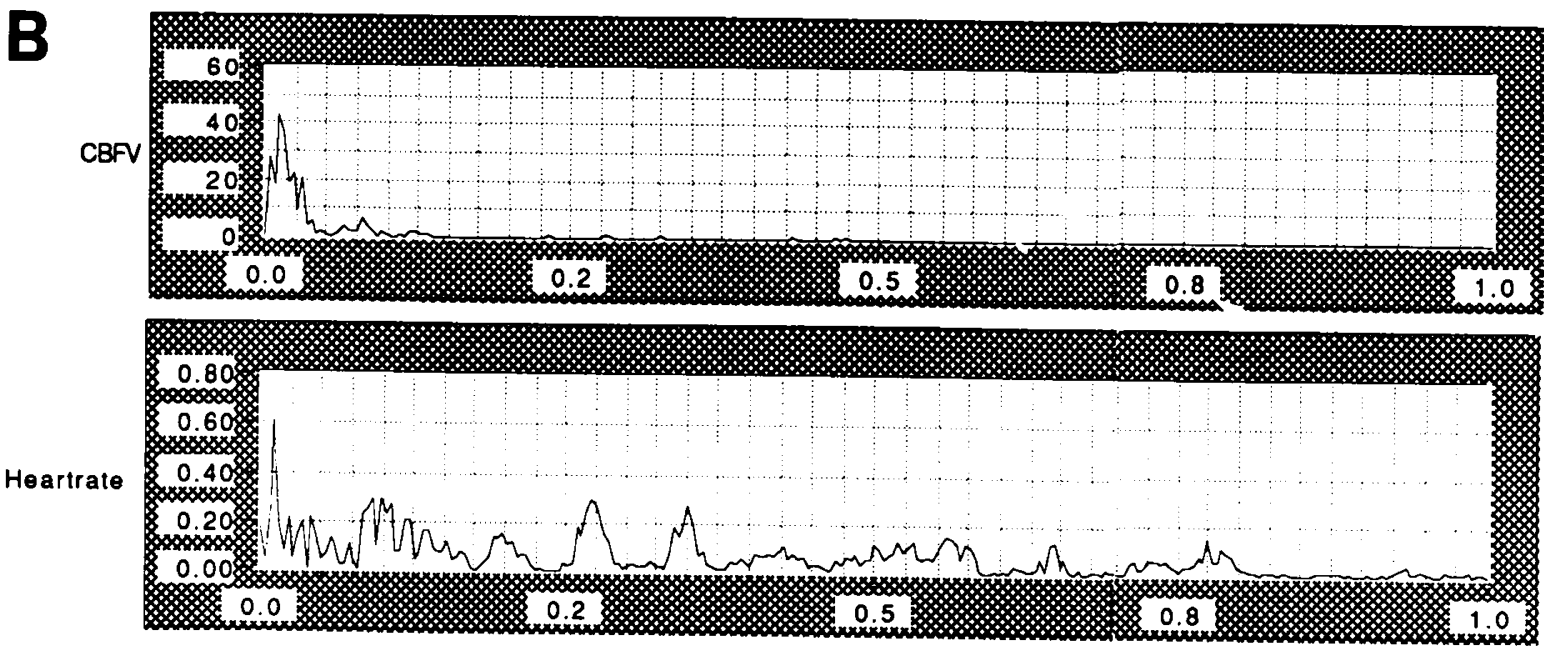

$\mathrm{Hz}$

Fig. 3. Fast Fourier transform analysis of the 4-min epochs shown in Figure 1. $A$, from the epoch collected in quiet sleep and $B$, from that collected in active sleep. Peak in the Fourier transform of the CBFV in $A$ between 0.04 and $0.06 \mathrm{~Hz}$ is not present in $B$.

apparent from visual inspection and a change of $24 \%$ is of similar magnitude to those induced by drugs such as indomethacin (18) which have been thought to be clinically important when persistent. The change in cycle frequency, although statistically significant, was very small and is unlikely to be of clinical importance. There was no difference in mean CBFV between sleep states in this study.

Results from Fourier analysis. Fourier analysis was used to resolve the frequencies of the variability present within the physiological signals. The results of subjecting the raw data present in Figure 1 to this type of analysis are shown in Figure $3, A$ and $B$. The heart rate variation was derived from the cycle lengths of the Doppler CBFV signal which had been "marked" using a foot-seeking algorithm (19). For clarity, only those frequencies occurring below $1 \mathrm{~Hz}(60 \mathrm{cycles} / \mathrm{min})$ are shown. The very slow oscillation in body temperature which is known to occur at $<0.02 \mathrm{~Hz}$ was confirmed and was reflected in the heart rate in both sleep states. No significant cycling in ICP was demonstrated, probably because the response time of the fontanometer was too slow to allow them to be recorded. There is a similar peak in CBFV at around $0.02 \mathrm{~Hz}$ in both the examples shown, whereas that seen in the recording made in quiet sleep (Figs. $1 A$ and $3 A$ ) shows a peak of 0.04 to $0.06 \mathrm{~Hz}$ which is not seen in active sleep, and does not correspond to change at a similar frequency in heart rate, temperature, or ICP. Analysis of all 167 recordings in this way showed that $33 / 66(50 \%)$ made in quiet sleep had a peak between 0.04 and $0.06 \mathrm{~Hz}$ compared to $35 / 101(34 \%)$ made in active sleep. This difference is significant ( $p=0.02$, Mann Whitney). The fast Fourier transform results thus confirm those obtained by the blind visual classification.

\section{DISCUSSION}

These data provide evidence that the CBFV of normal babies varies by about $25 \%$ during $1 \mathrm{~min}$, and that one component of this variability is a regular slow cycling at a frequency of 3 to 4 times/min. The frequency of the cycles are similar to those of Lundberg B waves (0.5-2 cycles/min) and of similar magnitude and frequency of fluctuations in CBFV seen in preterm babies $(8,9)$, normal adults, and head-injured patients $(10,11)$. Slow periodic cycling is superimposed on the regular rhythm of several other physiological parameters. Heart rate variation has been extensively studied and is known to vary both with a respiratory frequency and at lower frequencies. Respiratory sinus arrhythmia is more marked during quiet sleep in babies $(20,21)$ but it has 
been suggested that the slower cycles in heart rate associated with temperature control are less marked during quiet sleep (22).

The origin of Lundberg B waves in ICP is still in dispute. In Cheynes-Stokes respiration slow cyclical changes in ICP, blood pressure, and respiratory depth were all in synchrony (23), but in less severely pathological states changes in ICP were not in phase with other rhythmic variations (24). It seems unlikely that change in depth of respiration alone could have accounted for the changes seen in the CBFV of the babies; no clear slow cycling in respiration at the frequency of interest was seen on visual inspection of the recorded epochs or found on Fourier transform of the respiratory trace. Neither does it seem likely that the changes were merely reflecting change in heart rate, although spectral analysis of heart rate has previously shown a peak at $0.04 \mathrm{~Hz}$ thought to be due to fluctuations in peripheral vascular resistance mediated in part by the parasympathetic nervous system and which can be abolished by blockade of the reninangiotensin system (25). In the present study we confirmed this peak was present in babies, but the power of the resolved frequency from the changes seen in the cerebral circulation was greater than that occurring in the heart rate alone. However it would be of considerable interest to study the effect of pharmacological manipulation of the renin-angiotensin and sympathetic nervous systems on the slow cycles.

The sleep state changes in CBFV found in this study are in the opposite direction to those found in long-term variations of CBFV in ill adults and opposite to the influence of behavioral state on variability CBFV in preterm babies studied by Ramaekers (26). The methodology used in his study was to measure the area under the curve of each cardiac cycle and to express variability as the coefficient of variation of the area under the curve. Thus variability was studied, but not specifically slow cycling; we and others have shown that there are several components to the variability in CBFV (27), as indeed have been shown in heart rate and blood pressure variation. This means that our results could differ because a different type of variability was studied by Ramaekers et al. (26), for example respiratory-induced variability. In view of the fact that shorter recordings were used this is quite likely to have been the case, alternatively prematurity and ill health may affect the normal control mechanisms involved in the generation of slow cycling and account for the difference in our results. The cycling changes in CBFV almost certainly reflect changes in pial artery size occurring in response to their involvement in autoregulation (28). These changes have also been demonstrated as changes in cerebral blood volume with near infrared spectroscopy (29). The demonstration of changes occurring in normal healthy infants so soon after birth makes it likely that the waves represent autoregulatory processes occurring in the normal brain. The results remind us that it is important to take the behavioral state into account in work using Doppler and that the situation in health and disease may be very different, meaning that results obtained using drugs, for example, in one group cannot be applied to another.

\section{REFERENCES}

1. Sakai F, Meyer JS, Karacau I, Derman S, Yamamoto M 1980 Normal human sleep: regional cerebral haemodynamics. Ann Neurol 7:471-478

2. Mukhtar AI, Cowan FM, Stothers JK 1982 Cranial blood flow and blood pressure changes during sleep in the human neonate. Early Hum Dev 6: 59-64

3. Rahilly PM 1980 Effects of sleep state and feeding on cranial blood flow of the human neonate. Arch Dis Child 55:265-270
4. Greisen G, Hellstrom-Westas L, Lou H, Rosen I, Svenningsen N 1985 Sleepwaking shifts and cerebral blood flow in stable preterm infants. Pediatr Res 19:1156-1159

5. Jorch G, Huster T, Rabe H 1990 Dependency of Doppler parameters in the anterior cerebral artery on behavioural states in preterm and term neonates. Biol Neonate 58:79-86

6. Evans DH, Schlindwein FS, Levene MI 1989 An automatic system for capturing and processing ultrasonic Doppler signals. Clin Phys Physio Meas 10: 241-251

7. Fenton A, Evans DH, Levene MI 1990 On line cerebral blood flow velocity and blood pressure measurement in neonates: a new method. Arch Dis Child 65:11-14

8. Anthony MY, Levene MI, Evans DH 1991 Cyclical variations in cerebral blood flow velocity. Arch Dis Child 66:12-16

9. Coughtrey H, Rennie JM, Evans DH 1991 Postnatal evolution of slow variability in cerebral blood flow velocity. Arch Dis Child 67:412-415

10. Newell DW, Aaslid R, Stoos R, Reulen HJ 1992 The relationship of blood flow velocity fluctuations to intracranial pressure B waves. J Neurosurg 76 : 415-421

11. Mautner D, Dirnagl U, Haberl R, Schmiedek P, Garner C, Villringer A, Einhaupl KM 1989 B Waves in healthy persons. In: Hoff JT, Betz AL (eds) Intracranial Pressure VII. Springer-Verlag Berlin, pp 209-212

12. Lindqvist $A$, Oja R, Hellman O, Valimaki I 1983 Impact of thermal vasomotor control on the heart rate variability of newborn infants. Early Hum Dev 8: $37-47$

13. Prechtl HFR 1977 The neurological examination of the full term newborn infant, 2 nd ed. Clinics in Developmental Medicine, No. 63, SIMP Heinemann Medical, London

14. Nijhuis JC, Chester BM, Prechtl HFR 1984 Behavioural states of the human fetus In: Prechtl HFR (ed) Continuity of Neural Functions from Prenatal to Postnatal Life. Blackwell Scientific Publications, London, pp 65-78

15. Schlindwein FS, Smith MJ, Evans DH 1988 Spectral analysis of Doppler signals and computation of the normalised first moment in real time using a digital signal processor. Med Biol Eng Comput 26:228-232

16. Evans DH 1985 On the measurement of the velocity of blood flow over the cardiac cycle using Doppler ultrasound. Ultrasound Med Biol 11:735-741

17. Rochefort M, Rolfe P, Wilkinson A 1987 New fontanometer for continuous estimation of intracranial pressure in the newborn. Arch Dis Child 62: 152-155

18. Evans DH, Levene MI, Archer LNJ 1987 The effect of indomethacin on cerebral blood flow velocity in premature infants. Dev Med Child Neurol 29:254-262

19. Evans DH 1988 A pulse foot-seeking algorithm for Doppler ultrasound waveforms. Clin Phys Physiol Meas 9:267-271

20. Hathorn MKS 1987 Respiratory sinus arrhythmia in newborns. J Physiol 385: $1-12$

21. Harper RM 1978 Development of sinus arrhythmia during sleeping and waking states in normal infant. Sleep 1:33-48

22. Siasso B, Hodgman JE, Cabal L, Hon EH 1979 Cardiac and respiratory activity in relation to gestation and sleep states in newborn infants. Pediatr Res 13: 1163-1166

23. Hashimoto M, Higashi S, Kogure Y, Fuiji H, Tokuda K, Ito H, Yamamoto S 1989 Respiratory and cardiovascular oscillations during B waves. In: Hoff JT, Betz AL (eds) Intracranial Pressure VII. Springer-Verlag, Berlin, pp 217219

24. Higashi S, Yamamoto S, Hashimoto M, Fuiji H, Ito H, Kogure Y, Tokuda K 1989 The role of vasomotor center and adrenergic pathway in B waves. In: Hoff JT, Betz AL (eds) Intracranial Pressure VII. Springer-Verlag, Berlin, pp 220-224

25. Akselrod S, Gordon D, Ubel FA, Shannon DC, Barger AC, Cohen RJ 1981 Power spectrum analysis of heart rate fluctuations: a quantitative probe of beat to beat cardiovascular control. Science 213:220-222

26. Ramaekers V Th, Casaer P, Daniel H, Smet M, Marchal G 1989 The influence of behavioural states on cerebral blood flow velocity patterns in stable preterm infants. Early Hum Dev 29:229-246

27. Coughtrey H, Rennie JM, Evans DH, Cole TJ 1993 Factors associated with respiration induced variability in cerebral blood flow velocity. Arch Dis Child 68:312-316

28. Sayama I, Auer LM 1983 Oscillating cerebral blood volume: the origin of B waves. In: Ishii S, Nagai H, Brock M (eds) Intracranial Pressure V. SpringerVerlag, Berlin, pp 307-309

29. Livera LN, Wickramasinghe YABD, Spencer SA, Rolfe P, Thorniley MS 1992 Cyclical variations in cerebral blood volume. Arch Dis Child 67:62-63 